\title{
A PROJECTIVE ALGORITHM FOR STATIC OUTPUT FEEDBACK STABILIZATION
}

\author{
Kaiyang Yang*广, Robert Orsi* and John B. Moore*广 \\ * Department of Systems Engineering, The Australian \\ National University, Canberra ACT 0200, Australia. \\ † National ICT Australia Limited, Locked Bag 8001, \\ Canberra ACT 2601, Australia. \\ \{Kaiyang.Yang, Robert.Orsi, John.Moore\}@anu.edu.au
}

\begin{abstract}
This paper presents an algorithm for the static output feedback stabilization problem. The algorithm is iterative in nature and is based on alternating projection ideas. The main computational component of each iteration of the algorithm is a Schur matrix decomposition and the solution of a standard least squares problem. The paper contains computational results illustrating the effectiveness of the algorithm. Copyright (c) 2004 IFAC.
\end{abstract}

Keywords: alternating projections, stabilization, static output feedback.

\section{INTRODUCTION}

Consider the static output feedback (SOF) stabilization problem.

Problem 1. Given a linear time invariant (LTI) system

$$
\begin{aligned}
& \dot{x}=A x+B u \\
& y=C x
\end{aligned}
$$

where $x \in \mathbb{R}^{n}, u \in \mathbb{R}^{m}$, and $y \in \mathbb{R}^{p}$, find a static output feedback control law

$$
u=K y
$$

where $K \in \mathbb{R}^{m \times p}$ is a constant matrix such that the eigenvalues of the resulting closed-loop system matrix $A+B K C$ have non-positive real parts.

Problem 1 is not always solvable. Indeed, there are no testable necessary and sufficient conditions for the existence of a solution for Problem 1 (Syrmos et al., 1997).

Various numerical methods have been proposed for solving Problem 1. A comparison of such algo- rithms is given in (de Oliveira and Geromel, 1997). More recent work includes (El Ghaoui et al., 1997) and (Iwasaki, 1999). All the algorithms appearing in these papers attempt to solve the problem by trying to solve related non-convex problems involving linear matrix inequalities (LMIs).

In this paper we present a new algorithm for the SOF stabilization problem. We show the problem is equivalent to finding a point in the intersection of two closed sets. Our algorithm is iterative in nature and utilizes Schur's matrix decomposition in an alternating projection like scheme.

Other algorithms based on alternating projection ideas can be applied to the SOF problem, see in particular (Grigoriadis and Beran, 1999) and (Orsi et al., 2004). However, unlike these methods, our algorithm does not involve LMIs.

While the algorithm is often able to find solutions, like other existing algorithms, convergence to a solution is not guaranteed even if a solution exists. The ability to start the algorithm from different initial conditions and the ability to modify a cer- 
tain algorithm parameter increase the likelihood that a solution can be found.

The problem solved by our algorithm is a feasibility problem involving non-symmetric matrices. The idea of solving such problems via alternating projections that utilize Schur's decomposition originates in (Orsi and Yang, n.d.).

Finally we note that being able to solve the SOF problem enables one to solve other related problems. For example, finding a $K$ that places the closed loop eigenvalues in the set $\{z \in \mathbb{C} \mid \operatorname{Re}(z) \leq$ $-\alpha\}$ can be achieved by solving a SOF problem with $A$ replaced by $A+\alpha I$. ( $I$ denotes the identity matrix.) The reduced-order dynamic output feedback stabilization problem can also be solved via a system augmentation technique; see for example (Syrmos et al., 1997).

The structure of the paper is as follows. The remainder of this section lists some notation which is used in the rest of the paper. Section 2 introduces projections and how alternating projections can be used to find a point in the intersection of a finite number of closed (convex) sets. In order to motivate our solution methodology, in Section 3 we first consider a special case of the SOF problem in which the system and the output feedback matrix are symmetric. Section 4 considers the general non-symmetric problem and includes a description of the algorithm. Section 5 contains computational results. The paper ends with some concluding remarks.

Some notation: $\mathcal{S}^{r}$ is the set of real symmetric $r \times r$ matrices. $\operatorname{diag}(v)$ for $v \in \mathbb{C}^{r}$ denotes the $r \times r$ diagonal matrix whose $i$ 'th diagonal term is $v_{i} . \rho(Z)$ denotes the maximum of the real parts of the eigenvalues of the matrix $Z$. For $Z \in \mathbb{C}^{r \times s}$, $\operatorname{vec}(Z) \in \mathbb{C}^{r s}$ consists of the columns of $Z$ stacked below each other (Horn and Johnson, 1991). $Y \otimes Z$ denotes the Kronecker product of $Y$ and $Z$ (Horn and Johnson, 1991). For $Z \in \mathbb{C}^{r \times s}, \operatorname{Re}(Z) \in \mathbb{R}^{r \times s}$ and $\operatorname{Im}(Z) \in \mathbb{R}^{r \times s}$ denote respectively the real and imaginary parts of $Z ; Z=\operatorname{Re}(Z)+i \operatorname{Im}(Z)$.

\section{PROJECTIONS}

This section introduces projections and how alternating projections can be used to find a point in the intersection of a finite number of closed (convex) sets.

Let $x$ be an element in a Hilbert space $H$ and let $\mathcal{C}$ be a closed (possibly non-convex) subset of $H$. Any $c_{0} \in \mathcal{C}$ such that $\left\|x-c_{0}\right\| \leq\|x-c\|$ for all $c \in \mathcal{C}$ will be called a projection of $x$ onto $\mathcal{C}$. In the cases of interest here, namely that $H$ is a finite dimensional Hilbert space, there is always at least one such point for each $x$. If $\mathcal{C}$ is convex as well as closed then each $x$ has exactly one such minimum distance point. Any function $P_{\mathcal{C}}: H \rightarrow H$ will be called a projection operator (for $\mathcal{C}$ ) if for each $x \in H$,

$$
\begin{gathered}
P_{\mathcal{C}}(x) \in \mathcal{C} \text { and } \\
\left\|x-P_{\mathcal{C}}(x)\right\| \leq\|x-c\| \text { for all } c \in \mathcal{C} .
\end{gathered}
$$

A point in the intersection of a finite number of closed convex sets can be found via alternating projections (Brègman, 1965).

Theorem 2. Let $\mathcal{C}_{1}, \ldots, \mathcal{C}_{N}$ be closed convex sets in a real finite dimensional Hilbert space $H$. If $\bigcap_{i=1}^{N} \mathcal{C}_{i}$ is nonempty, then starting from an arbitrary initial value, the following sequence

$$
x_{i+1}=P_{\mathcal{C}_{\phi(i)}}\left(x_{i}\right) \text {, where } \phi(i)=(i \bmod N)+1 \text {, }
$$

converges to an element in $\bigcap_{i=1}^{N} \mathcal{C}_{i}$.

When one or more $\mathcal{C}_{i}$ 's are non-convex, Theorem 2 no longer applies and starting the algorithm of Theorem 2 from certain initial values may result in a sequence of points that does not converge to a solution of the problem. However, (Combettes and Trussell, 1990) suggests that alternating projections for problems with one or more non-convex sets converge locally; they will converge if the initial starting point is sufficiently close to a feasible point.

\section{THE SYMMETRIC PROBLEM}

In order to motivate our solution methodology, we first consider the following special case, the socalled symmetric case.

Problem 3. A LTI system of the following form

$$
\begin{aligned}
& \dot{x}=A x+B u, \quad A=A^{T}, \\
& y=B^{T} x,
\end{aligned}
$$

where $x \in \mathbb{R}^{n}, u \in \mathbb{R}^{m}$, and $y \in \mathbb{R}^{m}$, will be termed symmetric. For such a system, the symmetric SOF problem is to find $K \in \mathcal{S}^{m}$ such that $\rho\left(A+B K B^{T}\right) \leq 0$.

As the system is symmetric and as we restrict $\mathrm{K}$ to be symmetric, the symmetric SOF problem is equivalent to an LMI problem. Hence, if the problem is solvable, a numerical solution to the problem can be readily found using existing LMI algorithms, see for example (Vandenberghe and Boyd, 1996).

We now introduce a different solution method based on alternating projections. The main advantage of this new scheme will be that it can be extended to the general non-symmetric SOF problem. 
Let

$$
\begin{aligned}
\mathcal{L}=\left\{Z \in \mathcal{S}^{n} \mid Z=A+B K B^{T}\right. & \\
& \text { for some } \left.K \in \mathcal{S}^{m}\right\}
\end{aligned}
$$

and let $\mathcal{M}$ denote the negative semidefinite matrices,

$$
\mathcal{M}=\left\{Z \in \mathcal{S}^{n} \mid \rho(Z) \leq 0\right\} .
$$

The symmetric SOF problem can be stated as follows:

$$
\text { Find } X \in \mathcal{L} \cap \mathcal{M} \text {. }
$$

As the sets $\mathcal{L}$ and $\mathcal{M}$ are both convex and as projections onto these sets are readily calculated, a solution method is to alternatively project onto $\mathcal{L}$ and $\mathcal{M}$. Projection onto $\mathcal{L}$ involves solving a least squares problem. (The details of this projection are not used in the rest of the paper and are omitted.) Projection onto $\mathcal{M}$ is given by Theorem 5 below and is based on the following result of Hoffman and Wielandt.

Lemma 4. Suppose $Y, Z \in \mathcal{S}^{n}$ have eigenvalueeigenvector decompositions

$$
\begin{array}{ll}
Y=V D V^{T}, & D=\operatorname{diag}\left(\lambda_{1}^{Y}, \ldots, \lambda_{n}^{Y}\right), \\
Z=W E W^{T}, & E=\operatorname{diag}\left(\lambda_{1}^{Z}, \ldots, \lambda_{n}^{Z}\right),
\end{array}
$$

where $V, W \in \mathbb{R}^{n \times n}$ are orthogonal and $\lambda_{1}^{Y} \geq$ $\ldots \geq \lambda_{n}^{Y}$ and $\lambda_{1}^{Z} \geq \ldots \geq \lambda_{n}^{Z}$. Then

$$
\|D-E\| \leq\|Y-Z\|,
$$

where $\|\cdot\|$ denotes the Frobenius norm.

Proof. See for example (Horn and Johnson, 1985, Corollary 6.3.8).

Theorem 5. Given $Y \in \mathcal{S}^{n}$, let $Y=V D V^{T}$ be an eigenvalue-eigenvector decomposition of $Y$ with $D=\operatorname{diag}\left(\lambda_{1}, \ldots, \lambda_{n}\right)$. If $\bar{D}=\operatorname{diag}\left(\min \left\{0, \lambda_{1}\right\}, \ldots\right.$, $\left.\min \left\{0, \lambda_{n}\right\}\right)$, then $P_{\mathcal{M}}(Y):=V \bar{D} V^{T}$ is the best approximant in $\mathcal{M}$ to $Y$ in the Frobenius norm.

Proof. Let $Y$ be as in the theorem statement. As $P_{\mathcal{M}}(Y) \in \mathcal{M}$, it remains to show

$$
\left\|Y-P_{\mathcal{M}}(Y)\right\| \leq\|Y-Z\| \text { for all } Z \in \mathcal{M} .
$$

Without loss of generality,suppose the eigenvalues of $Y$ are ordered, i.e., $\lambda_{1} \geq \ldots \geq \lambda_{n}$. Similarly, for $Z \in \mathcal{M}$, let $Z=W E W^{T}$ be an eigenvalueeigenvector decomposition with $\lambda_{1}^{Z} \geq \ldots \geq \lambda_{n}^{Z}$. Using the fact that the Frobenius norm is orthogonally invariant, we have

$$
\begin{aligned}
\left\|Y-P_{\mathcal{M}}(Y)\right\| & =\left\|V(D-\bar{D}) V^{T}\right\| \\
& =\|D-\bar{D}\| .
\end{aligned}
$$

By Lemma 4, the theorem follows if we can show

$$
\|D-\bar{D}\| \leq\|D-E\| .
$$

To show this, note that

$$
\|D-E\|^{2}=\sum_{k}\left(D_{k k}-E_{k k}\right)^{2} .
$$

A similar equality holds for $\|D-\bar{D}\|^{2}$. Hence (1) holds if we can show, given any $d \in \mathbb{R}$,

$$
(d-\min \{0, d\})^{2} \leq(d-e)^{2} \text { for all } e \leq 0 .
$$

This inequality readily follows by considering the two possible cases, $d \leq 0$ and $d>0$.

Note that to calculate $P_{\mathcal{M}}(Y)$ we keep the original orthogonal matrix $V$ and simply modify the diagonal matrix $D$ to $\bar{D}$. The fact that $V$ remains unchanged will be used to motivate our solution method for the general non-symmetric case.

\section{THE GENERAL NON-SYMMETRIC PROBLEM}

In this section, $\mathbb{C}^{n \times n}$ will be regarded as a Hilbert space with inner product

$$
\langle Y, Z\rangle=\operatorname{tr}\left(Y Z^{*}\right)=\sum_{i, j} y_{i j} \bar{z}_{i j} .
$$

The associated norm is the Frobenius norm $\|Z\|$ $=\langle Z, Z\rangle^{\frac{1}{2}}$.

Consider again Problem 1, i.e., the general nonsymmetric SOF problem. Let $\mathcal{L}$ now denote the set of all possible closed-loop system matrices,

$$
\begin{aligned}
\mathcal{L}=\left\{Z \in \mathbb{R}^{n \times n} \mid Z=A+B K C\right. & \\
& \text { for some } \left.K \in \mathbb{R}^{m \times p}\right\},
\end{aligned}
$$

and let $\mathcal{M}$ now denote the set of matrices with eigenvalues in the left half plane,

$$
\mathcal{M}=\left\{Z \in \mathbb{C}^{n \times n} \mid \rho(Z) \leq 0\right\} .
$$

The non-symmetric problem can be stated as:

$$
\text { Find } X \in \mathcal{L} \cap \mathcal{M} \text {. }
$$

The set $\mathcal{L}$ is again an affine subspace and hence convex. However, $\mathcal{M}$ is no longer a convex set. For example, the matrices

$$
Z_{1}=\left(\begin{array}{rr}
-1 & 4 \\
0 & -1
\end{array}\right) \quad \text { and } \quad Z_{2}=\left(\begin{array}{rr}
-1 & 0 \\
4 & -1
\end{array}\right)
$$

are elements of $\mathcal{M}$, however, the convex combination $\left(Z_{1}+Z_{2}\right) / 2$ has eigenvalues $\{-3,1\}$ and hence is not in $\mathcal{M}$.

The general non-symmetric problem is much harder to solve than the symmetric problem. There are two reasons for this. The first is the non-convexity of $\mathcal{M}$; alternating projections are no longer guaranteed to converge. The second, even more important issue is that it is not clear how to project onto $\mathcal{M}$. That is, given a point $Z$, it is not clear how to find a point in $\mathcal{M}$ of minimal distance to $Z$.

Motivated by the symmetric case, we propose the following map as a substitute for a projection map onto $\mathcal{M}$. 
Before proceeding, recall Schur's result (Horn and Johnson, 1985).

Theorem 6 . Given $Z \in \mathbb{C}^{n \times n}$ with eigenvalues $\lambda_{1}, \ldots, \lambda_{n}$ in any prescribed order, there is a unitary matrix $V \in \mathbb{C}^{n \times n}$ and an upper triangular matrix $T \in \mathbb{C}^{n \times n}$ such that

$$
Z=V T V^{*}
$$

and $T_{k k}=\lambda_{k}, k=1, \ldots, n$.

Definition 7 . For any $V \in \mathbb{C}^{n \times n}$ unitary and any $T \in \mathbb{C}^{n \times n}$ upper triangular, define

$$
P_{\mathcal{M}}(V, T)=V \bar{T} V^{*}
$$

where

$\bar{T}_{k l}= \begin{cases}\min \left\{0, \operatorname{Re}\left(T_{k k}\right)\right\}+i \operatorname{Im}\left(T_{k k}\right), & \text { if } k=l, \\ T_{k l}, & \text { otherwise. }\end{cases}$

A given $Z \in \mathbb{C}^{n \times n}$ may have a non-unique Schur decomposition and $Z=V_{1} T_{1} V_{1}^{*}=V_{2} T_{2} V_{2}^{*}$ does not imply $P_{\mathcal{M}}\left(V_{1}, T_{1}\right)=P_{\mathcal{M}}\left(V_{2}, T_{2}\right)$. Hence, $P_{\mathcal{M}}$ may give different points for different Schur decompositions of the same matrix. Despite this, the following result shows that different Schur decompositions lead to points in $\mathcal{M}$ of equal distance from the original matrix.

Theorem 8. Suppose $Z=V_{1} T_{1} V_{1}^{*}=V_{2} T_{2} V_{2}^{*}$ where $V_{1}, V_{2} \in \mathbb{C}^{n \times n}$ are unitary and $T_{1}, T_{2} \in$ $\mathbb{C}^{n \times n}$ are upper triangular. Then

$$
\left\|P_{\mathcal{M}}\left(V_{1}, T_{1}\right)-Z\right\|=\left\|P_{\mathcal{M}}\left(V_{2}, T_{2}\right)-Z\right\| .
$$

Proof. Suppose $Z=V T V^{*}$ where $V$ is unitary and $T$ is upper triangular. If $\bar{T}$ is the matrix given by Definition 7 , then by the unitary invariance of the Frobenius norm

$$
\left\|P_{\mathcal{M}}(V, T)-Z\right\|=\|\bar{T}-T\| .
$$

From Definition 7 , if $\lambda_{1}, \ldots, \lambda_{n}$ are the eigenvalues of $Z$, then

$$
\|\bar{T}-T\|^{2}=\sum_{i}\left(\min \left\{0, \operatorname{Re}\left(\lambda_{i}\right)\right\}-\operatorname{Re}\left(\lambda_{i}\right)\right)^{2} .
$$

The result follows by noting that the right hand side of (2) only depends on the eigenvalues of $Z$. In particular this value would be exactly the same for any other Schur decomposition of $Z$.

For the next theorem, we define

$$
\begin{array}{r}
\mathcal{T}=\left\{T \in \mathbb{C}^{n \times n} \mid T\right. \text { is upper triangular } \\
\text { and } \rho(T) \leq 0\} .
\end{array}
$$

Note, Theorem 6 implies

$$
\begin{aligned}
& \mathcal{M}=\left\{Z \in \mathbb{C}^{n \times n} \mid Z=V T V^{*}\right. \\
& \quad \text { for some unitary } V \text { and some } T \in \mathcal{T}\} .
\end{aligned}
$$

Theorem 9. Suppose $Z=V T V^{*} \in \mathbb{C}^{n \times n}$ with $V$ unitary and $T$ upper triangular. Then $P_{\mathcal{M}}(V, T)$ satisfies

$$
\left\|P_{\mathcal{M}}(V, T)-Z\right\| \leq\left\|V \tilde{T} V^{*}-Z\right\| \text { for all } \tilde{T} \in \mathcal{T} \text {. }
$$

Proof. Let $\tilde{T}$ be a matrix in $\mathcal{T}$. The unitary invariance of the Frobenius norm implies the result will be established if we can show

$$
\|\bar{T}-T\| \leq\|\tilde{T}-T\|
$$

where $\bar{T}$ is the matrix given in Definition 7. As both $\tilde{T}$ and $T$ are upper triangular, it follows that

$$
\|\tilde{T}-T\|^{2}=\sum_{k=1}^{n}\left|\tilde{T}_{k k}-T_{k k}\right|^{2}+\sum_{k<l}\left|\tilde{T}_{k l}-T_{k l}\right|^{2} .
$$

Using similar reasoning to that used in the proof of Theorem 5 , it follows that $\|\bar{T}-T\|^{2}$ is less than or equal to the first summation on the right hand side of the equality in (4). Hence, (3) holds.

Finally, note that the map of Definition 7 is not a projection. For example if $Z=V T V^{*}$ where

$V=\left(\begin{array}{cc}0.8165 & -0.5774 \\ 0.5774 & 0.8165\end{array}\right), T=\left(\begin{array}{cc}2.4142 & 1.0000 \\ 0 & -0.4142\end{array}\right)$,

then it is readily verified that if $Y=\left(\begin{array}{ll}0 & 1 \\ 0 & 0\end{array}\right)$ then $\left\|Z-P_{\mathcal{M}}(V, T)\right\| \not\|Z-Y\|$.

Projection onto $\mathcal{L}$ involves solving a standard least squares problem.

Lemma 10. The projection of $X \in \mathbb{C}^{n \times n}$ onto $\mathcal{L}$ is given by $P_{\mathcal{L}}(X)=A+B K C$ where $K$ is a solution of the least squares problem

$\underset{K \in \mathbb{R}^{m \times p}}{\arg \min }\left\|\left(C^{T} \otimes B\right) \operatorname{vec}(K)-\operatorname{vec}(\operatorname{Re}(X)-A)\right\|_{2}$.

Here $\|\cdot\|_{2}$ denotes the standard vector 2 norm.

Proof. We would like to find $K \in \mathbb{R}^{m \times p}$ that minimizes

$$
\|X-(A+B K C)\|^{2} .
$$

As $A, B$ and $C$ are real matrices, it follows that (5) equals

$$
\|\operatorname{Re}(X)-(A+B K C)\|^{2}+\|\operatorname{Im}(X)\|^{2},
$$

and hence that the problem is equivalent to minimizing the first term in (6).

The result now follows by noting that for any $Z \in \mathbb{C}^{n \times n},\|Z\|=\|\operatorname{vec}(Z)\|_{2}$, and that for any (appropriately sized) matrices $P, Q$ and $R$, $\operatorname{vec}(P Q R)=\left(R^{T} \otimes P\right) \operatorname{vec}(Q)$ (Horn and Johnson, 1991). 
Here is our algorithm for the SOF stabilization problem.

Algorithm:

Problem Data. $A \in \mathbb{R}^{n \times n}, B \in \mathbb{R}^{n \times m}, C \in$ $\mathbb{R}^{p \times n}$.

Initialization. Choose a randomly generated $Y \in \mathbb{R}^{n \times n}$. For example, draw each entry of $Y$ from a normal distribution of zero mean and variance 1 .

repeat

1. Calculate a Schur decomposition of $Y$ : $Y=V T V^{*}$.

2. $X:=P_{\mathcal{M}}(V, T)$.

3. $Y:=P_{\mathcal{L}}(X)$. until $\rho(Y) \leq 0$.

While convergence of the above algorithm is not guaranteed, as will be demonstrated in the next section, it does indeed often converge to a solution.

Numerical experiments show that the performance of the algorithm can actually be improved by using the following slightly modified version of $P_{\mathcal{M}}$ which depends on a parameter $\gamma \in \mathbb{R}$.

Definition 11. Let $\gamma \in \mathbb{R}$ be non-positive. For any $V \in \mathbb{C}^{n \times n}$ unitary and any $T \in \mathbb{C}^{n \times n}$ upper triangular, define

$$
P_{\mathcal{M}}^{\gamma}(V, T)=V \bar{T} V^{*}
$$

where

$\bar{T}_{k l}= \begin{cases}\min \left\{\gamma, \operatorname{Re}\left(T_{k k}\right)\right\} & +i \operatorname{Im}\left(T_{k k}\right), \\ T_{k l}, & \text { if } k=l \text { and } \operatorname{Re}\left(T_{k k}\right) \geq 0,\end{cases}$

$P_{\mathcal{M}}^{\gamma}$ shifts the real parts of the unstable eigenvalues to $\gamma(\leqslant 0)$ rather than to 0 . (If $\gamma=0$ then $P_{\mathcal{M}}^{0}$ is just $P_{\mathcal{M}}$.) As we will see in the numerical results section, choosing $\gamma<0$ can significantly reduce the number of iterations required to converge to a solution.

Finally we note (without proof) that if $A=A^{T}$, $C=B^{T}$, and the initial condition $Y$ is chosen symmetric, then the algorithm with $\gamma$ set to zero reduces to the one outlined in Section 3 for the symmetric problem.

\section{COMPUTATIONAL RESULTS}

This section presents computational results for some randomly generated problems and a particular problem from the literature.

The algorithm was implemented in Matlab 6.5 and all results were obtained using a $3.06 \mathrm{GHz}$ Pentium 4 machine, with $1 \mathrm{~Gb}$ of memory, running Windows XP Professional.

\subsection{Randomly generated examples}

This subsection contains results for some randomly generated problems. In order to guarantee that the problems have solutions, we choose the system dimensions so that they satisfy Kimura's generic stabilization condition (Kimura, 1975): $n<m+p$. In particular, we take $n=6, m=4$ and $p=3$. For each random problem, each of the elements of A, B and C were drawn from a normal distribution of zero mean and variance 1 .

A 1000 randomly generated problems were constructed. Table 1 shows how effective the algorithm is in solving these problems using various values of $\gamma$. Shown are the percentage of problem that converged within certain iteration ranges and the average CPU time. As can be seen, the algorithm performs best with $\gamma<0$. Performance is roughly equally good for each negative $\gamma$ and, for these $\gamma$ 's, solutions were found in about 50\% of cases. Though not reported here, even smaller values of $\gamma$ produce similar results to $\gamma=-3$ and $\gamma=-5$. (Values down to $\gamma=-20$ were tested.)

Table 1. A comparison of performance for different values of $\gamma . i$ denotes the number of iterations, ' $\mathrm{NC}$ ' denotes nonconvergence after 1000 iterations, and $T$ denotes the average convergence time in $\mathrm{CPU}$ seconds for the problems that converge within 1000 iterations.

\begin{tabular}{ccccc} 
No. of iterations & $\gamma=0$ & $\gamma=-1$ & $\gamma=-3$ & $\gamma=-5$ \\
\hline $1 \leq i \leq 10$ & $1 \%$ & $22 \%$ & $28 \%$ & $30 \%$ \\
$10<i \leq 100$ & $8 \%$ & $19 \%$ & $15 \%$ & $13 \%$ \\
$100<i \leq 1000$ & $25 \%$ & $7 \%$ & $5 \%$ & $4 \%$ \\
$\mathrm{NC}$ & $66 \%$ & $52 \%$ & $52 \%$ & $53 \%$ \\
\hline \hline $\mathrm{T}$ & 0.17 & 0.03 & 0.03 & 0.03 \\
\hline
\end{tabular}

Figure 1 shows how the success rate improves if we try to solve each problem using up to 10 different initial conditions.

Fig. 1. A comparison of performance for different values of $\gamma$ using up to 10 initial conditions.

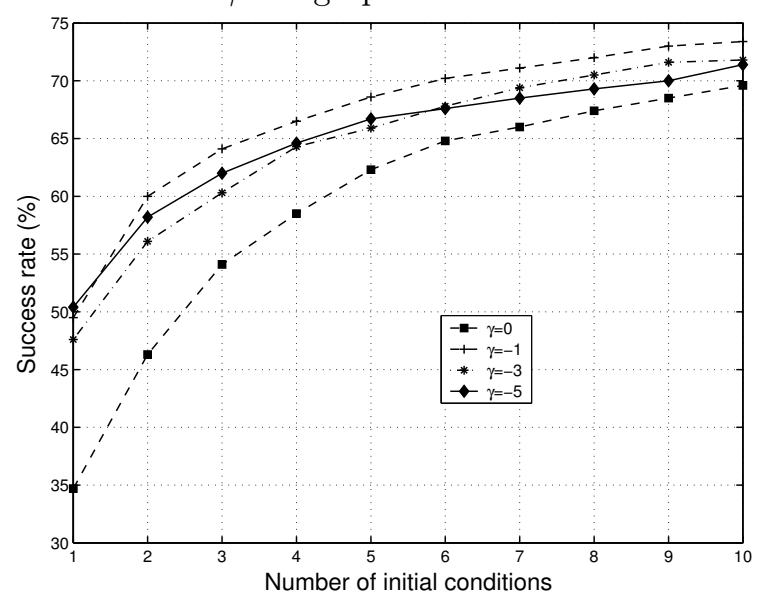

For all $\gamma$ 's, solutions were found in about $70 \%$ of cases. The average total solution time for the 
problems that were successfully solved was 0.68 CPU seconds.

\subsection{A problem from the literature}

The following problem taken from (Keel et al., 1988) appears frequently in the literature. The system considered is the nominal linearized model of a helicopter:

$$
\begin{aligned}
& A=\left(\begin{array}{rrrr}
-0.0366 & 0.0271 & 0.0188 & -0.4555 \\
0.0482 & -1.0100 & 0.0024 & -4.0208 \\
0.1002 & 0.3681 & -0.7070 & 1.4200 \\
0.0000 & 0.0000 & 1.0000 & 0.0000
\end{array}\right), \\
& B=\left(\begin{array}{rr}
0.4422 & 0.1761 \\
3.5446 & -7.5922 \\
-5.5200 & 4.4900 \\
0.0000 & 0.0000
\end{array}\right), C=\left(\begin{array}{l}
0 \\
1 \\
0 \\
0
\end{array}\right)^{T} .
\end{aligned}
$$

In this problem we wish to place the closed loop eigenvalues in the set $\{z \in \mathbb{C} \mid \operatorname{Re}(z) \leq-\alpha\}$ with $\alpha=0.1$. To achieve this, we apply the algorithm with $A$ replaced by $A+\alpha I$. Regarding the choice of the parameter $\gamma$, a number of different values are selected. When $\gamma \geq-17$, the algorithm is not always convergent. However, when $\gamma \leq-18$, for example $-18,-19,-20, \ldots$, the algorithm appears to be always convergent. Typically the algorithm converges within 1000 iterations, with computational time under $0.7 \mathrm{CPU}$ seconds. A particular solution is

$$
K=\left(\begin{array}{ll}
0.0939 & 1.1127
\end{array}\right)^{T}
$$

for which $A+B K C$ has eigenvalues $\{-0.1440$, $-9.3716,-0.1765 \pm i 0.7909\}$.

\section{CONCLUSIONS}

This paper has proposed a novel projective algorithm for the static output feedback stabilization problem. As for other algorithms for this problem, convergence to a solution is not guaranteed. Even so the algorithm can be useful in practice. The ability to start the algorithm from different initial conditions and the ability to modify a certain algorithm parameter increasing the likelihood that problems can be solved.

\section{ACKNOWLEDGEMENTS}

The second and third authors acknowledge the support of the Australian Research Council through grants DP0450539 and A00105829.
National ICT Australia is funded by the Australian Department of Communications, Information Technology and the Arts and the Australian Research Council through Backing Australia's Ability and the ICT Centre of Excellence Program.

\section{REFERENCES}

Brègman, L. M. (1965). The method of successive projection for finding a common point of convex sets. Soviet Mathematics 6(3), 688692.

Combettes, P. L. and H. J. Trussell (1990). Method of successive projections for finding a common point of sets in metric spaces. $J$. Optim. Theory Appl. 67, 487-507.

de Oliveira, M. C. and J. C. Geromel (1997). Numerical comparison of output feedback design methods. In: Proc. American Control Conference. Vol. 1. pp. 72-76.

El Ghaoui, L., F. Oustry and M. Ait Rami (1997). A cone complementarity linearization algorithm for static output-feedback and related problems. IEEE Trans. Automat. Control 42(8), 1171-1176.

Grigoriadis, K. M. and E. B. Beran (1999). Alternating projection algorithms for linear matrix inequalities problems with rank constraints. Advances on Linear Matrix Inequality in Control, pp. 251-267. L. El Ghaoui and S,-I. Niculescu, Eds. SIAM.

Horn, R. A. and C. A. Johnson (1985). Matrix Analysis. Cambridge University Press.

Horn, R. A. and C. A. Johnson (1991). Topics in Matrix Analysis. Cambridge University Press.

Iwasaki, T. (1999). The dual iteration for fixedorder control. IEEE Trans. Automat. Control 44(4), 783-788.

Keel, L. H., S. P. Bhattacharyya and J. W. Howze (1988). Robust control with structured perturbation. IEEE Trans. Automat. Control 33(1), 68-78.

Kimura, H. (1975). Pole assignment by gain output feedback. IEEE Trans. Automat. Control 20, 509-516.

Orsi, R. and K. Yang (n.d.). Numerical methods for solving inverse eigenvalue problems for nonnegative matrices. In preparation.

Orsi, R., U. Helmke and J. B. Moore (2004). A Newton-like method for solving rank constrained linear matrix inequalities. To appear in Proc. 43rd IEEE Conf. on Decision and Control, Paradise Island, Bahamas.

Syrmos, V. L., C. T. Abdallah, P. Dorato and K. Grigoriadis (1997). Static output feeback - a survey. Automatica 33(2), 125-137.

Vandenberghe, L. and S. Boyd (1996). Semidefinite programming. SIAM 38(1), 49-95. 